\title{
Auroral all-sky camera calibration
}

\author{
F. Sigernes ${ }^{1, *}$, S. E. Holmen ${ }^{1, *}$, D. Biles ${ }^{2}$, H. Bjørklund ${ }^{1}$, X. Chen ${ }^{1, *}$, M. Dyrland ${ }^{1, *}$, D. A. Lorentzen ${ }^{1, *}$, L. Baddeley ${ }^{1, *}$, \\ T. Trondsen ${ }^{3}$, U. Brändström ${ }^{4}$, E. Trondsen ${ }^{5}$, B. Lybekk ${ }^{5}$, J. Moen ${ }^{5}$, S. Chernouss ${ }^{6}$, and C. S. Deehr ${ }^{7}$ \\ ${ }^{1}$ University Centre in Svalbard, Longyearbyen, Norway \\ ${ }^{2}$ Magnetosphere Ionosphere Research Lab, University of New Hampshire, USA \\ ${ }^{3}$ Keo Scientific Ltd, Calgary, Alberta, Canada \\ ${ }^{4}$ Swedish Institute of Space Physics, Kiruna, Sweden \\ ${ }^{5}$ Department of Physics, University of Oslo, Oslo, Norway \\ ${ }^{6}$ Polar Geophysical Institute, Murmansk Region, Apatity, Russia \\ ${ }^{7}$ Geophysical Institute, University of Alaska, Fairbanks, USA \\ *also at: Birkeland Centre for Space Science, University of Bergen, Bergen, Norway
}

Correspondence to: F. Sigernes (freds@unis.no)

Received: 8 August 2014 - Published in Geosci. Instrum. Method. Data Syst. Discuss.: 1 September 2014 Revised: 28 October 2014 - Accepted: 13 November 2014 - Published: 10 December 2014

\begin{abstract}
A two-step procedure to calibrate the spectral sensitivity to visible light of auroral all-sky cameras is outlined. Center pixel response is obtained by the use of a Lambertian surface and a standard $45 \mathrm{~W}$ tungsten lamp. Screen brightness is regulated by the distance between the lamp and the screen. All-sky flat-field correction is carried out with a $1 \mathrm{~m}$ diameter integrating sphere. A transparent Lexan dome at the exit port of the sphere is used to simulate observing conditions at the Kjell Henriksen Observatory (KHO). A certified portable low brightness source from Keo Scientific Ltd was used to test the procedure. Transfer lamp certificates in units of Rayleigh per Ångstrøm (R/Å) are found to be within a relative error of $2 \%$. An all-sky camera flat-field correction method is presented with only 6 required coefficients per channel.
\end{abstract}

\section{Introduction}

During the last decades, numerous ground-based all-sky cameras have been installed in both hemispheres to monitor aurora and airglow. In the northern hemisphere, the fields of view of these cameras overlap to cover large sections of the aurora oval (cf. Akasofu, 1964). The desire to, for example, estimate and compare auroral hemispherical power as measured by satellites (cf. Zhang and Paxton, 2008) requires unified and accurate calibration routines (Brändström et al.,
2012) to quantify the radiance in photometric units (Hunten et al., 1956). This paper presents a two-step procedure to calibrate to sensitivity the all-sky cameras at the Kjell Henriksen Observatory (KHO).

\section{Experimental setup}

The calibration tools are shown in Fig. 1. The fixed imaging compact spectrograph (FICS) is mounted on a heightadjustable table. The table can be moved on rails towards the Lambertian screen. The entrance optics of FICS is a $22^{\circ}$ field-of-view-fused silica fibre bundle. The spectrograph is made by ORIEL (model 77443). It uses a concave holographic grating (230 grooves $/ \mathrm{mm})$. The nominal spectral range is $4000-11000 \AA$, and the bandpass is approximately $80 \AA$ with the $100 \mu \mathrm{m}$ wide entrance slit. The detector is a 16 bit dynamic range thermoelectric cooled CCD camera from the company Andor (model DU 420A-OE). Our main calibration source, the $45 \mathrm{~W}$ tungsten lamp from Oriel ( $\mathrm{s} / \mathrm{n} 7$ 1867), is also mounted on the table. The lamp is a traceable National Institute of Standards (NIST) source. The lamp certificate is listed in Table 1. Both the Lambertian screen (SRT99-180) and the $1 \mathrm{~m}$ diameter integrating sphere (CSTM-LR40) are made by the company Labsphere. Note that in Fig. 1, the spectrograph is set up to measure the output of the integrating sphere. 


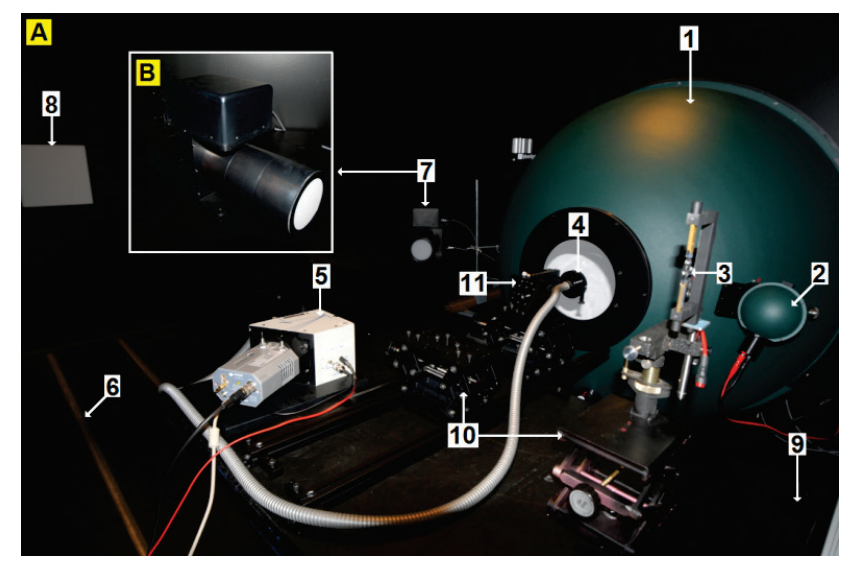

Figure 1. Experimental setup at the UNIS calibration lab. (a) (1) Labsphere 1m diameter low light level integrating sphere, (2) source sphere with tungsten lamp, (3) 45W tungsten lamp, (4) FICS fiber bundle probe, (5) FICS, (6) rail road, (7) Keo Alcor-RC low light source, (8) Lambertian screen, (9) height adjustable table on rails, (10) table jacks and (11) rotary probe mount. (b) Keo Alcor-RC low light source.

Table 1. Oriel $45 \mathrm{~W}$ tungsten lamp certificate ( $\mathrm{s} / \mathrm{n} 7-1867)$. The spectral irradiance values are measured at a distance of $z_{0}=0.5 \mathrm{~m}$.

\begin{tabular}{|c|c|c|}
\hline $\begin{array}{l}\text { Wavelength } \\
\lambda[\AA]\end{array}$ & $\begin{array}{r}\text { Irradiance } \\
M_{0}(\lambda) \\
{\left[\mathrm{mW} \mathrm{m}^{-2} \mathrm{~nm}^{-1}\right]}\end{array}$ & $\begin{array}{r}\text { Irradiance } \\
M_{0}(\lambda) \\
{\left[\# \text { photons cm } \mathrm{cm}^{-2} \mathrm{~s}^{-1} \AA^{-1}\right]}\end{array}$ \\
\hline 4000 & 0.79670 & $1.60221 \times 10^{10}$ \\
\hline 4500 & 1.71388 & $3.87755 \times 10^{10}$ \\
\hline 5000 & 2.99143 & $7.51994 \times 10^{10}$ \\
\hline 5550 & 4.65315 & $1.29839 \times 10^{11}$ \\
\hline 6000 & 6.04915 & $1.82478 \times 10^{11}$ \\
\hline 6546 & 7.64049 & $2.51456 \times 10^{11}$ \\
\hline 7000 & 8.76666 & $3.08530 \times 10^{11}$ \\
\hline 8000 & 11.1985 & $4.50416 \times 10^{11}$ \\
\hline
\end{tabular}

The integrating sphere is modified by including a transparent dome at the exit port and a baffle to block light from the source sphere. A sketch of the modifications is shown in Fig. 2. The dome is made of the same material (Lexan) and thickness $(5 \mathrm{~mm})$ as the domes at the KHO. The all-sky cameras should be inserted into the dome in order to fill the total field of view of $180^{\circ}$. The baffle acts as a moon blocker. The net result is that observational and calibration conditions are the same for all optical instruments housed at the KHO.

The FICS is sensitivity-calibrated by the use of the Lambertian screen and the $45 \mathrm{~W}$ tungsten lamp. The distance and angle between the screen and the lamp regulate the brightness of the screen. This well-known method of calibrating narrow field-of-view instruments is described in detail by Sigernes et al. (2007).

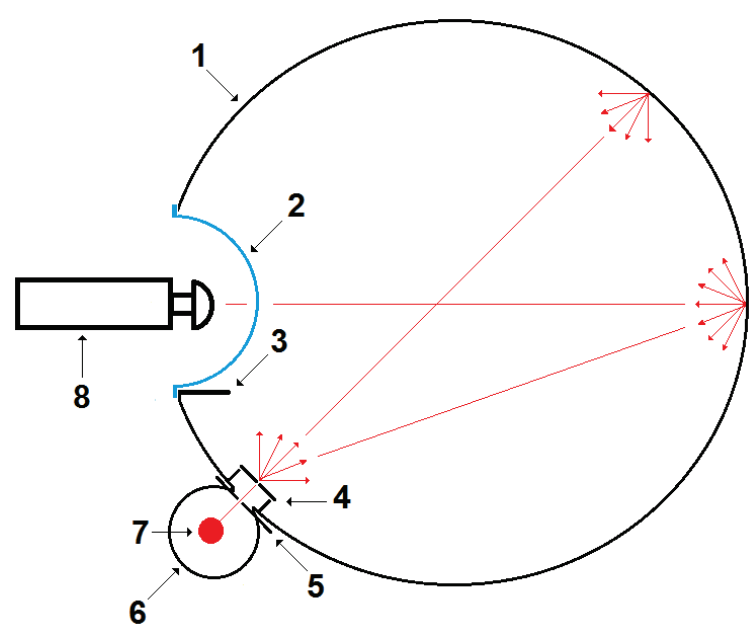

Figure 2. Sketch of modification to the $1 \mathrm{~m}$ diameter integrating sphere: (1) Labsphere CSTM-LR-40, (2) transparent Lexan dome, (3) baffle, (4) transparent diffusor, (5) adjustable aperture, (6) source sphere, (7) tungsten lamp and (8) instrument with all-sky lens. Red arrows and lines indicate the effect of multiple scattering inside the sphere.

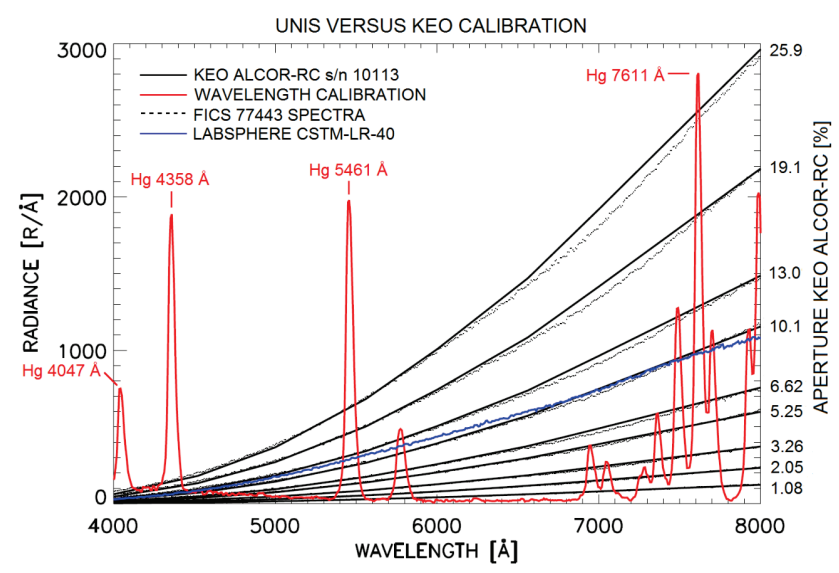

Figure 3. Absolute and wavelength calibration of the FICS. The line spectrum in red is from a mercury vapor lamp supplied by Edmund Optics Ltd (SN K60-908). The solid black spectra are from the Keo Alcor-RC certificate. The corresponding black dotted lines are spectra measured by the FICS. The spectrum of the integrating sphere is plotted in blue.

\section{Test and tuning of calibration tools}

A mobile low light source made by the company Keo Scientific is used to test the new calibration method. The head unit of the Keo Alcor-RC low brightness source (s/n 10113) is visible in Fig. 1. It contains a $100 \mathrm{~W}$ tungsten lamp, aperture wheels and diffusors to attenuate the brightness of the opal output surface. The source is certified by the National Research Council of Canada (NRC).

The FICS fiber probe is mounted head-on to the center of the output surface of the Keo-Alcor-RC source. The field of 
Table 2. The difference in spectral calibration between UNIS - FICS and NRC of the Keo Alcor-RC low brightness source as a function of aperture. All numbers are in units of $\%$.

\begin{tabular}{llllllllll}
\hline Keo Alcor - RC aperture & 25.9 & 19.1 & 13.0 & 10.1 & 6.62 & 5.24 & 3.26 & 2.05 & 1.08 \\
\hline Difference & 2.00 & 1.56 & 1.41 & 0.40 & 1.42 & 0.04 & 0.90 & 0.26 & 1.96 \\
\hline
\end{tabular}

Table 3. Fish-eye mapping functions. $F$ is the effective focal length of the lens, and $\theta$ is the angle to the optical axis.

\begin{tabular}{lr}
\hline Type & Fish-eye mapping function \\
\hline Linear scaled & $R=f \times \theta$ \\
Orthographic & $R=f \times \sin (\theta)$ \\
Equal area & $R=2 \times f \times \sin (\theta / 2)$ \\
Stereographic & $R=2 \times f \times \tan (\theta / 2)$ \\
\hline
\end{tabular}

view of the probe is then within a $1 \mathrm{~cm}$ diameter spot size of the diffuse surface. The setup is compatible to the spectral irradiance measured by Gaertner (2013). Figure 3 shows the measured FICS spectra and the corresponding certified spectra from NRC as a function of aperture setting. The Keo Alcor-RC aperture is given in units of percentage. $100 \%$ is maximum brightness, while $10 \%$ means that the brightness is one tenth of maximum.

The percent of error between the measured integrated FICS spectra and the NRC certificate is used to quantify the relative difference in the calibrations. The results are listed in Table 2 as a function of Keo aperture. Note that the relative errors are less than or equal to $2 \%$.

The next step in the test is to move the FICS fiber probe to the center of the Labsphere integrating sphere output port and tune the aperture of the source sphere down to a level that corresponds to the one tenth aperture brightness of the Alcor$\mathrm{RC}$ source. The spectral radiance is then below the threshold of $\sim 1 \mathrm{kR} / \AA$, which is close to the intensity range of magnitude for auroras and airglow as indicated by the blue-colored spectrum in Fig. 3.

\section{Basic camera equations}

This section derives the equations needed for calibration of multiple wavelength filtered all-sky cameras. The experimental setup is as sketched in Fig. 2 using the integrating sphere as source. The type of filters used depends on center wavelength $\lambda_{\mathrm{c}}$, bandpass BP and transmission $T$. The most traditional one is the Fabry-Perot filter design, where parallel transparent glass plates create interference due to multiple reflections between the plates.

The raw data counts of the camera at pixel position $(x, y)$ are given as an integral over wavelength:

$u=\int B(\lambda) \cdot S(\lambda) \mathrm{d} \lambda,[$ cts $]$,

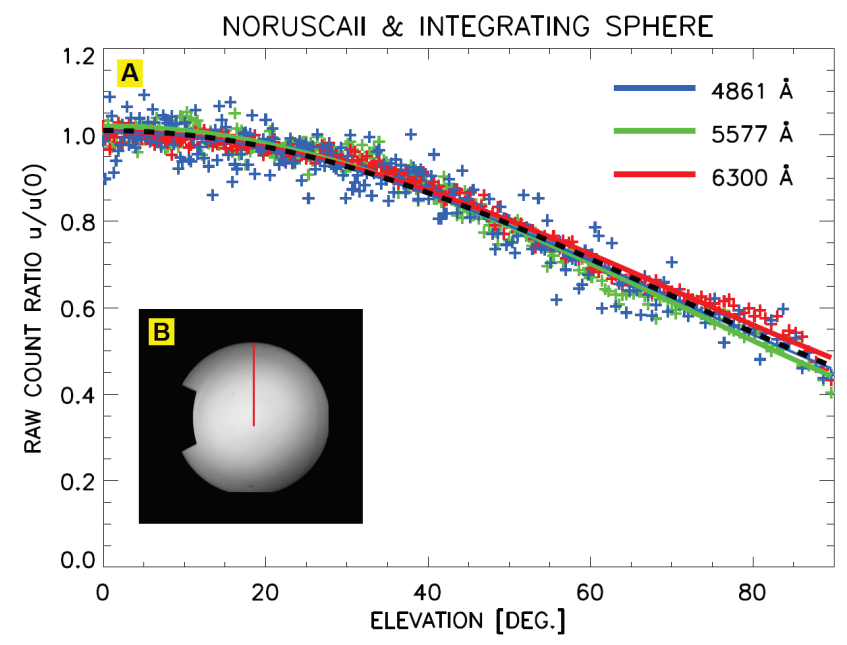

Figure 4. NORUSCA II all-sky camera data of integrating sphere by Labsphere (CSTM-LR-40). (a) Blue, red and green crosses are raw count ratios as function of elevation $\theta$ for wavelengths 4861 , 5577 and $6300 \AA$, respectively. Exposure time is $1 \mathrm{sec}$ and detector gain is 100 . Center image count is defined as $u(\theta=0)$. Solid colored lines are corresponding functional fits to the data. The dotted black line is the average fit over the wavelength. (b) Grayscale image of integrating the sphere at the center wavelength $5577 \AA$. The red-colored line marks center-to-edge coordinates used to obtain the count ratios.

Table 4. NORUSCA II all-sky camera center value calibration factors.

\begin{tabular}{lrrrr}
\hline & $\begin{array}{r}\text { Wavelength } \\
\lambda_{\mathrm{c}}[\AA]\end{array}$ & $\begin{array}{r}\text { Emission } \\
\text { species }\end{array}$ & $\begin{array}{r}\text { Bandpass } \\
\mathrm{BP}[\AA]\end{array}$ & $\begin{array}{r}\text { Calibration factor } \\
B\left(\lambda_{\mathrm{c}}\right) \cdot \mathrm{BP} / u(0) \\
{\left[\mathrm{R} \mathrm{cts}^{-1}\right]}\end{array}$ \\
\hline 1 & 4278 & $\mathrm{~N}_{2}^{+}$ & 54.4 & 110.2 \\
2 & 4500 & Background & 57.3 & 88.7 \\
3 & 4709 & $\mathrm{~N}_{2}^{+}$ & 59.9 & 60.1 \\
4 & 4861 & $\mathrm{H}_{\beta}$ & 61.9 & 44.4 \\
5 & 5002 & $\mathrm{NII}$ & 63.7 & 38.1 \\
6 & 5577 & {$[\mathrm{OI}]$} & 71.0 & 25.1 \\
7 & 5680 & $\mathrm{NII}$ & 72.3 & 23.7 \\
8 & 5890 & $\mathrm{NaI}$ & 75.0 & 22.3 \\
9 & 6300 & {$[\mathrm{OI}]$} & 80.2 & 18.8 \\
10 & 6364 & {$[\mathrm{OI}]$} & 81.0 & 17.7 \\
11 & 6563 & $\mathrm{H}_{\alpha}$ & 83.5 & 16.6 \\
12 & 6624 & $\mathrm{~N}_{2} 1 \mathrm{P}(6-3)$ & 84.3 & 15.7 \\
13 & 6705 & $\mathrm{~N}_{2} 1 \mathrm{P}(5-2)$ & 85.3 & 14.4 \\
14 & 6764 & $\mathrm{~N}_{2}$ & 86.1 & 14.1 \\
15 & 7000 & Background & 89.1 & 12.4 \\
\hline
\end{tabular}


where $S$ is defined as the spectral responsivity and $B$ is the source spectrum in absolute units. The wavelength dependency of the spectral responsivity is assumed to be proportional to the transmission of the filter:

$S(\lambda) \approx \varepsilon \cdot T(\lambda) \cdot\left[\operatorname{cts~R}^{-1}\right]$.

If the source $B$, lens transmissions and detector sensitivity vary slowly in the wavelength interval $\Delta \lambda$, then Eq. (1) becomes

$u=B\left(\lambda_{\mathrm{c}}\right) \cdot \varepsilon \cdot \int T(\lambda) \mathrm{d} \lambda=B\left(\lambda_{\mathrm{c}}\right) \cdot \varepsilon \cdot A$,

where $A$ is the area of the filter transmission curve. It is assumed that $T$ is narrow and triangular in shape. Then

$A=\int T(\lambda) \mathrm{d} \lambda \approx T_{m} \cdot \mathrm{BP}$,

where $T_{m}$ is the peak transmission of the filter at $\lambda=\lambda_{\mathrm{c}}$. Furthermore, the spectral radiance of a discrete auroral emission line at wavelength $\lambda_{c}$ is defined as

$J_{\mathrm{a}}(\lambda) \equiv J \cdot \delta\left(\lambda-\lambda_{\mathrm{c}}\right)[\mathrm{R} / \AA]$,

where $\delta$ is the Kronecker delta. The number of auroral raw data counts is then

$$
\begin{aligned}
& u_{\mathrm{a}}=\int J_{\mathrm{a}}(\lambda) \cdot S(\lambda) \mathrm{d} \lambda=\int J \cdot \delta\left(\lambda-\lambda_{\mathrm{c}}\right) \cdot \varepsilon \cdot T(\lambda) d \lambda \\
& =J \cdot \varepsilon \cdot \int T(\lambda) \cdot \delta\left(\lambda-\lambda_{\mathrm{c}}\right) \mathrm{d} \lambda=J \cdot \varepsilon \cdot T_{\mathrm{m}} .
\end{aligned}
$$

Finally, from Eqs. (3), (4) and (6),

$J=u_{\mathrm{a}} \times\left[\frac{B\left(\lambda_{\mathrm{c}}\right) \cdot \mathrm{BP}}{u}\right] \cdot[\mathrm{R}]$.

Note that Eq. (7) is only valid when the transmission profile of the filter is narrow and triangular.

The $(B / u)$ factor for each pixel in the all-sky image must be examined further. Let us introduce the radial center pixel distance, $R$, of a point in the image plane defined as

$R \equiv \sqrt{\left(x-x_{\mathrm{c}}\right)^{2}+\left(y-y_{\mathrm{c}}\right)^{2}}$,

where $\left(x_{\mathrm{c}}, y_{\mathrm{c}}\right)$ are the center pixel coordinates. The relation between $R$ and the zenith angle $\theta$ is known as the radial mapping function. Table 3 lists typical fish-eye mapping functions. In a more general form, Kumler and Bauer (2000) suggested that circular image fish-eye lenses have mapping functions equal to

$R=k_{1} \cdot f \cdot \sin \left(k_{2} \cdot \theta\right) .[\mathrm{mm}]$.

For the hyperspectral all-sky camera at KHO named NORUSCA II (Sigernes et al., 2012), the coefficients are $f=$ $3.5 \mathrm{~mm}, k_{1}=1.2$ and $k_{2}=0.83$, using known star positions as input data.
The radiance $B$ of the integrating sphere is per definition uniform in all directions of $\theta$, and $(x, y)$ points with equal $R$ should, due to symmetry, have the same raw data count rate of $u$. As a consequence, it is useful to transform our $(x, y)$ coordinates to $(R, \theta)$ coordinates with Eqs. (8) and (9). It must be emphasized that the above assumption is for an ideal conditioned sphere with no deterioration of the inside coating (Barium sulfate) over time. A functional fit to the data for each wavelength channel may then be found as

$u=u(\theta) \approx u(0) \cdot\left[a_{0} \cos \left(a_{1} \cdot \theta\right)+a_{2}\right]$.

A 3rd degree polynomial fit may also be used. The final form of Eq. (7) becomes

$J=u_{\mathrm{a}} \times\left[\frac{B\left(\lambda_{\mathrm{c}}\right) \cdot \mathrm{BP}}{u(0)}\right] \times\left[\frac{1}{a_{0} \cos \left(a_{1} \cdot \theta\right)+a_{2}}\right]$.

Note that the left bracket in Eq. (11) only requires center $(\theta=0)$ values $u$, while the right bracket describes the offaxis $(\theta>0)$ behavior of the camera. It is known as flat-field correction of image $u_{\mathrm{a}}$.

Based on the above equations, we propose the calibration to be undertaken in two steps. The first step is to find the coefficients $a_{0}, a_{1}$ and $a_{2}$ by using the integrating sphere. The second step is to measure the center pixel area counts of $u(0)$ by using the Lambertian screen setup instead of the integrating sphere. The screen to lamp distance $z$ is very useful to regulate screen brightness to the same order of magnitude as that expected during sampling of the aurora. The exposure time and gain settings are identical for both the calibration and normal dark-sky operation of the cameras. The latter also cancels out any effect due to nonlinear behavior of count levels versus exposure time. The spectral radiance of the screen is given as

$B(\lambda)=\left(\frac{4 \rho}{10^{6}}\right) \times M_{0}(\lambda) \times\left(\frac{z_{0}}{z}\right)^{2} \times \cos \alpha \cdot[\mathrm{R} / \AA]$.

$M_{0}(\lambda)$ is the known irradiance (certificate) of the lamp in units of [\#photons $\mathrm{cm}^{-2} \mathrm{~s}^{-1} \AA^{-1}$ ], initially obtained at a distance of $z_{o}=0.5 \mathrm{~m}$ (see Table 1 ). The diffuse reflectance factor $\rho$ of the screen is nearly constant $(\rho=0.98)$ throughout the visible and near infrared regions of the spectrum. The angle $\alpha$ is between the screen and the tungsten lamp $(\alpha=0)$.

\section{Results}

Three $1 \mathrm{sec}$ exposures at center wavelengths 4861,5577 and $6300 \AA$ of the integrating sphere were used to obtain the raw count ratio $u(\theta) / u(0)$ for the NORUSCA II camera. The results are shown in Fig. 4. A functional LMFIT in IDL (Interactive Data Language) based on the Levenberg-Marquardt algorithm results in coefficients $a_{0}=0.38, a_{1}=1.29$ and $a_{2}=0.63$ (see Eq. 10). Note that in our case there is no significant difference in shape and level of the raw count ratio between the three wavelength channels. This leads to the 
conclusion that the off-axis effect of the NORUSCA II camera is the same for all wavelengths across the visible spectrum. Or in other words, flat-field correction is independent of wavelength. All parameters in the right bracket of Eq. (11) are now found.

The center pixel calibration factors of the NORUSCA II camera $\left[B\left(\lambda_{\mathrm{c}}\right) \cdot \mathrm{BP} / u(0)\right]$ were found by the method described by Sigernes et al. (2007). 15 wavelength bands were selected to cover the most prominent auroral emissions within the spectral range of the camera. Table 4 lists the origin of the emissions and the corresponding calibration factors.

The net result is that the camera is sensitivity-calibrated and flat-field corrected with only six parameters per channel. For each pixel we obtain the radial center distance $R$ using Eq. (8). Two parameters, $k_{1}$ and $k_{2}$, are used in Eq. (9) to calculate the elevation $\theta$. Three more parameters, $a_{0}, a_{1}$ and $a_{2}$ in Eq. (10), are then used to correct for off-axis effects. The sixth parameter is the center pixel calibration factor $\left[B\left(\lambda_{c}\right) \cdot \mathrm{BP} / u(0)\right]$ of Eq. (11).

The above procedure is fast to compute and ideal for realtime display of all-sky calibrated data.

\section{Conclusions}

A two-step method to calibrate and flat-field correct an allsky camera is outlined. The center pixel spectral sensitivity is obtained and tested by a traditional method including a flat Lambertian screen and a $45 \mathrm{~W}$ tungsten lamp. Flat-field correction or off-axis response is conducted by the use of a modified $1 \mathrm{~m}$ diameter integrating sphere. The net result is that it is sufficient with only six parameters per channel to calibrate an all-sky camera.
Acknowledgements. We wish to thank Arnold A. Gaertner at NRC for assistance and checking our calibration equations. This work is financially supported by the Research Council of Norway through the project named: Norwegian and Russian Upper Atmosphere Co-operation on Svalbard part 2 \# 196173/S30 (NORUSCA2).

Edited by: S. Szalai

\section{References}

Akasofu, S. I.: The latitudinal shift of the auroral belt, J. Atmosph. Terr. Phys., 26, 1167-1174, 1964.

Brändström, B. U. E., Enell, C.-F., Widell, O., Hanson, T., Whiter, D., Mäkinen, S., Mikhaylova, D., Axelsson, K., Sigernes, F., Gulbrandsen, N., Schlatter, N. M., Gjendem, A. G., Cai, L., Reistad, J. P., Daae, M., Demissie, T. D., Andalsvik, Y. L., Roberts, O., Poluyanov, S., and Chernouss, S.: Results from the intercalibration of optical low light calibration sources 2011, Geosci. Instrum. Method. Data Syst., 1, 43-51, 2012.

Gaertner, A.: Spectral radiance of low brightness source serial number 10113, Calibration report no. PAR-2012-3049, Institute for National Measurement Standards, National Research Council Canada, 1-11, 2013

Hunten, D. M., Roach, F. E., and Chamberlain, J. W.: A photometric unit for the airglow and aurora, J. Atmosph. Terr. Phys., 8, 345346, 1956.

Kumler, J. J. and Bauer, M. L.: Fish-eye lens design and their relative performance, Proc. SPIE 4093, Current Developments in Lens Design and Optical Systems Engineering, 360 (24 October 2000), doi:10.1117/12.405226, 2000.

Sigernes, F., Holmes, J. M., Dyrland, M., Lorentzen, D.A., Chernouss, S. A., Svenøe, T., Moen, J., and Deehr, C. S.: Absolute calibration of optical devices with a small field of view, J. Opt. Technol., 74, 669-674, 2007.

Sigernes, F., Ivanov, Y., Chernouss, S., Trondsen, T., Roldugin, A., Fedorenko, Y., Kozelov, B., Kirillov, A., Kornilov, I., Safargaleev, V., Holmen, S., Dyrland, M., Lorentzen, L., and Baddeley, L.: Hyperspectral all-sky imaging of auroras, Opt. Express, 20, 27650-27660, 2012.

Zhang, Y. and Paxton, L. J.: An empirical Kp-dependent global auroral model based on TIMED/GUVI data, J. Atmos. Solar-Terr. Phys., 70, 1231-1242, 2008. 